\section{Drug interactions}

\author{
Per Hartvig Honoré
}

\section{DRUG-NON-DRUG INTERACTIONS}

- Identifying and handling possible interactions is the most common pharmacokinetics and pharmacodynamics related task for many pharmacists in pharmacies and in the hospital ward.

- Handling interactions requires both deep knowledge and the competence to act when necessary but, more importantly, without frightening the patient unnecessarily.

Drugs may interact with the vehicle or the container, with food, with herbal medicines and with other drugs. Some of these are absolute such as the physical interactions, while others are relative with incidences rates ranging from very high to almost none.

Physical drug interactions caused by an incorrect $\mathrm{pH}$, oxidising or reducing agents in the solution, exposure to light and nonreversible adsorption to container walls or excipients should be thoroughly investigated before marketing. Such interactions can easily be measured and the competence of the pharmacist may further guarantee that they do not occur.

Food interactions can be divided into food-drug interactions and drug-food interactions. Interaction with food causing lowered or delayed absorption is rare and mostly occurs with metal-ion chelate complex drugs such as tetracyclines, oxiquinolones and some cytotoxics. The second type of interaction includes situations where extended drug treatment induces possibly detrimental physiological and even pathophysiological changes. Insulin producing drugs, corticosteroids, antipsychotics and antidepressants may increase appetite leading to weight gain, while drugs causing nausea (eg, opioids) have the opposite effect. Antibiotic drugs may disturb mucosal function and the uptake of vitamin K. Folic acid absorption is lowered by phenytoin.

Hyperglycaemia can be caused by opioids, antipsychotics, sulphonamides,

Correspondence to Dr Per Hartvig Honoré, Department of Drug Design and Pharmacology, Faculty of Health and Medical Sciences, University of Copenhagen, Universitetsparken 2, 2100 Copenhagen, Denmark; peh@sund.ku.dk antiepileptic drugs and warfarin, and in some cases may develop into diabetes. On the other hand, hypoglycaemia can be seen after a patient takes acetyl salicylic acid or $\beta$-receptor blockers. Acetyl salicylic acid and colchicine for gout may decrease plasma lipid concentrations, while opioids, corticosteroids, growth hormone and vitamin D may raise plasma lipid concentrations after lengthy use. This type of interaction may cause serious risks but can be avoided by monitoring.

The possible interactions of herbal medicines with drugs is a huge issue, as little is known about the components of such medicines, their potency to induce or block metabolic enzymes or their interactions with many drugs. Some herbal remedies such as St John's wort have known risks if taken together with numerous medicines, while the risks posed by others are unpredictable.

\section{DRUG-DRUG INTERACTIONS}

Drug-drug interactions are very common and many thousands are described in standard prescribing reference books. However, there are few clinically relevant or significant drug-drug reactions. This is extremely important. Three types of drug-drug interactions need to be discussed with respect to clinical situations:

- The good: therapeutically useful (agonist-antagonist) interactions. In many clinical situations drugs interacting with each other are used for the benefit of the patient, such as antidotes administered after overdoses, drugs used to combat heart effects when reversing neuromuscular blockade by atropine after anaesthesia, leucovorin rescue given after a high methotrexate dose, etc.

- The awful: potentially harmful interactions which should be remembered and identified at an early stage.

- The only ugly: interactions of no or little clinical significance which constitute the vast majority and should be identified as being low risk.

It is not possible to remember all relevant drug-drug interactions or to look them up in reference manuals or computer databases due to time constraints in the clinical situation. However, one should memorise the more common and harmful drug-drug interactions, particularly those where one component has a small therapeutic window, as with warfarin, digoxin, tricyclic antidepressants, aminoglycosides, antiepileptic drugs such as phenytoin and carbamazepine, antiarrhythmics, AIDS drugs and ciclosporin.

Patients with co-morbidities, the elderly and those with impaired cardiac, liver or renal function should be assessed before a drug is prescribed or administered. If the pharmacist has time, a trustworthy reference source or medicine information centre can be consulted. Otherwise and in an emergency, pharmacokinetic reasoning can help. Knowledge of the chemical properties of similar drugs from the same class may allow some insight into absorption from or transport through the gastrointestinal tract, and whether metabolism might include enzyme induction or inhibition as well as competition for excretion via the kidneys. However, the conclusions drawn must be verified as soon as possible. Finally you must always ask yourself whether the effect could be caused by the drug itself-for example, a side effect, insufficient effect of the drug or disease breakthrough.

The contribution in this issue of the journal entitled 'Drug interactions with rifampicin-well known but not considered?' by Dr Dorothea Strobach reflects the current conflict in the assessment of drug-drug interactions. Numerous drug interactions with rifampicin with different risks are mentioned in the literature and are tabulated in interaction lists and in databases and are easily forwarded to the responsible physician. However, the interactions may be well known and have been experienced by the physician and the risk may not be relevant. Therefore the pharmacist may be worried while the physician is not. Should all these rifampicin interactions be considered harmful and patients exposed to a 'rifampicin interaction test'?

\section{Competing interests None.}

Provenance and peer review Commissioned; internally peer reviewed.

To cite Hartvig Honoré P. Eur J Hosp Pharm 2014;21:73.

Eur J Hosp Pharm 2014;21:73. doi:10.1136/ejhpharm-2013-000388 